\title{
CROSSPOINT COMPLEXITY OF SPARSE CROSSBAR CONCENTRATORS*
}

\author{
A. Yavuz Oruç and H. M. Huang \\ Electrical Engineering Department \\ University of Maryland \\ College Park, MD 20742
}

April 1995

\begin{abstract}
A sparse crossbar $(n, m, c)$-concentrator is a bipartite graph with $n$ inputs and $m$ outputs in which any $c$ or fewer inputs can be matched with an equal number of outputs, where $c$ is called its capacity. Sparse crossbar concentrators play a key role in multiplexing signals in communication systems and constructing permutation and generalized connection networks. In this paper we present a number of new results on the crosspoint complexity of such concentrators. First, we describe an $(n, m, m)$ concentrator construction, called a fat-and-slim crossbar whose crosspoint complexity matches Nakamura-Masson's lower bound for any given $n$ and $m$, thereby removing a restriction on the number of inputs and number of outputs imposed by Masson's binomial concentrator construction. Second, we present a $(2 m, m)$-concentrator with crosspoint complexity matching Nakamura-Masson's lower bound and with fanout which is nearly the half of the fanout of the binomial concentrator. Third, we derive a new lower bound on the crosspoint complexity of sparse crossbar $(n, m, c)$-concentrators. This bound closely follows Nakamura-Masson's lower bound, but unlike that bound, it is a simple rational function of $n, m$ and $c$, and hence, can be computed very easily. We show that this bound is attainable when $n-m \leq c \leq \sqrt{m}$. Finally, we give an extension of this bound to cascades of sparse crossbar concentrators.
\end{abstract}

Key Words: Bipartite graph, concentrator, sparse crossbar, crosspoint complexity, binomial network.

\footnotetext{
*This work is supported in part by the Graduate Studies and Research Board at the University of Maryland, and in part by the National Science Foundation under Grant No. NCR-9405539. A preliminary version of this work was presented at the 1994 Information Sciences and Systems Conference at Princeton University, Princeton, New Jersey.
} 


\section{Introduction}

Given an $m \times n$ binary matrix, suppose we wish to determine if, for every $c$ columns of this matrix, there exist $c$ rows such that the intersection of every column in the given set of $c$ columns with a distinct row among those rows contains a " 1 " entry. For example, in the $6 \times 6$ matrix

$\begin{array}{llllll}1 & 1 & 1 & 0 & 0 & 0 \\ 1 & 0 & 0 & 1 & 1 & 0 \\ 0 & 1 & 0 & 1 & 0 & 1 \\ 0 & 0 & 1 & 0 & 1 & 1\end{array}$

the intersections of columns $1,2,3,4$ with rows $2,1,4,3$ in the given order, all contain " 1 " entries. It can be verified that the stated condition applies to all four columns of this matrix, whereas in the $4 \times 6$ matrix

$\begin{array}{llllll}1 & 1 & 1 & 0 & 0 & 0 \\ 1 & 0 & 0 & 1 & 1 & 1 \\ 0 & 1 & 0 & 1 & 0 & 1 \\ 0 & 0 & 1 & 0 & 1 & 0\end{array}$

the columns $1,2,4$ and 6 fail to meet this condition.

This interesting problem about binary matrices can also be posed as a matching question in bipartite graphs, and is closely related to the behavior of a switching device, called a sparse crossbar concentrator as follows. The columns in an $m \times n$ binary matrix represents the inputs of such a device, the rows represents its outputs, and the " 1 " entries correspond to contacts or crosspoints between the inputs and outputs. The condition that the intersections of every $c$ columns with some $c$ rows contain "1" entries characterizes the concentrator's ability to connect any $c$ of its inputs to some $c$ of its outputs. Any sparse crossbar whose columns meet this property will be called a sparse crossbar $(n, m, c)$-concentrator, where $c$ is called its capacity. Such devices play a central role in subscriber loops to multiplex low rate channels onto higher speed transmission trunks or remote carriers[12]. They are also used in the construction of more powerful connectors such as permutation networks and generalized connectors $[3,5,7]$.

In this paper we will focus on the crosspoint complexity of sparse crossbar concentrators and crosspoint complexity of cascades of such concentrators. We will also 
be concerned with the construction of sparse crossbar concentrators that meet the predicted crosspoint complexities. Needles to say, it is of theoretical interest to determine the crospoint complexity of a sparse crossbar concentrator. The problem has the flavor of a quintessential complexity question: With how little can one get away? More importantly, however, the crosspoint complexity of a concentrator has a significant bearing on designing cost-efficient concentrators, since the number of crosspoints used in a concentrator construction determines, to a large extent, the degree of coupling between its inputs and outputs. Extensive coupling between inputs and outputs can make the implementation of concentrators difficult on programmable logic arrays (PLAs and PALs) and custom VLSI circuits.

A number of results have been reported on the crosspoint complexity of concentrators. Pinsker proved that there exists an $(n, m, m)$-concentrator (henceforth to be called an $(n, m)$-concentrator) with at most $29 n$ crosspoints [11]. Explicit constructions of $(n, m, c)$-concentrators with $O(n)$ crosspoints were given by Margulis [8] and others $[4,1]$ for any $c, 1 \leq c \leq m$. While these constructions rely on $O(n)$ crosspoints, they are obtained by cascading $O(\log n)$ sparse crossbars together.

In another direction, Masson [9] and Nakamura and Masson [10] studied the crosspoint complexity of sparse crossbar concentrators. They derived lower bounds on the number of crosspoints in sparse crossbar concentrators and showed that, in certain cases, these bounds are tight. While their bound for full capacity $(c=m)$ concentrators is easy to compute, to determine their lower bound for bounded capacity $(c<m)$ concentrators, one must solve a polynomial whose degree depends on the capacity of the concentrator in question.

In this paper we extend these results. First, we describe an $(n, m)$-concentrator construction, called a fat-and-slim crossbar, whose crosspoint complexity matches Nakamura-Masson's lower bound for any given $n$ and $m$, thereby removing the restriction on the choices of number of inputs and outputs imposed by Masson's binomial network. Second, we present a $(2 m, m)$-concentrator whose crosspoint complexity also matches the same lower bound but with nearly half the fanout of the first construction. Third, we derive a new lower bound on the crosspoint complexity of sparse crossbar $(n, m, c)$-concentrators. This bound closely follows Nakamura-Masson's lower bound, 
Inputs

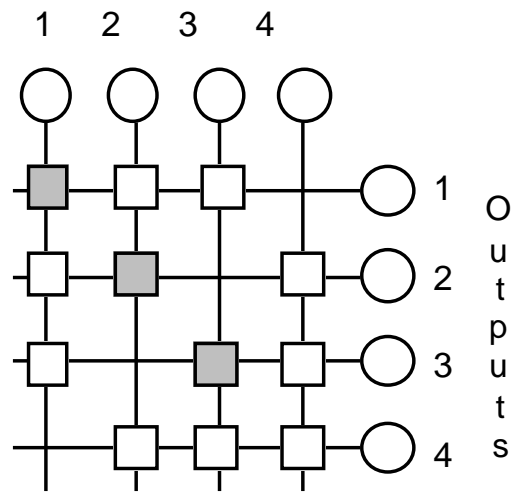

(a) $n=4, m=4, v=3$
Inputs

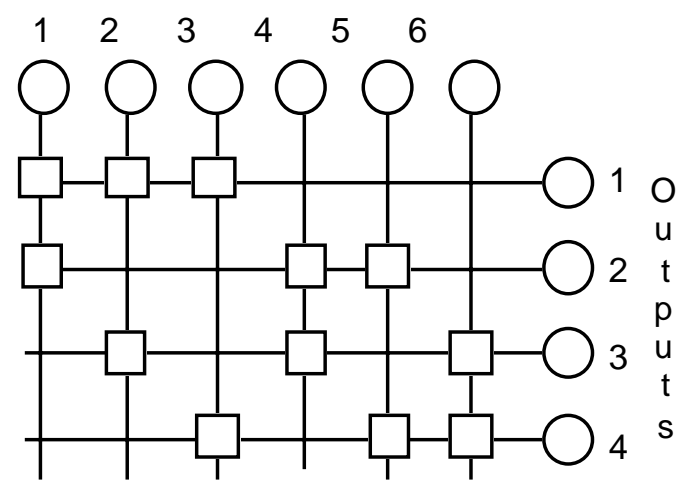

(b) $n=6, m=4, v=2$

Fig. 1: Binomial networks with four outputs.

but unlike that bound, it is very easy to compute. Next, we describe a sparse crossbar $(n, m, c)$-concentrator whose crosspoint complexity resides within a factor of two of this new lower bound for any $c, n-m \leq c \leq \sqrt{m}$. Finally, we derive a lower bound for the crosspoint complexity of any $(n, m, c)$ concentrator that is constructed by cascading a fixed number of sparse crossbar concentrators.

\section{Problem Formulation and Approach}

Formally, an $(n, m, c)$-concentrator is a directed acyclic graph $\mathcal{G}=(I, O, E)$, with a set of $n$ inputs $(I)$, a set of $m$ outputs $(O)$, and a set of edges $(E)$ such that there exist edge-disjoint paths between any $c$ or fewer inputs and an equal number of outputs, where $c$ is called its capacity. $\mathcal{G}$ is called a full capacity concentrator when $c=m$, and it is called a bounded capacity concentrator, otherwise. The edges in $E$ are called the crosspoints of $\mathcal{G}$. The number of crosspoints of $\mathcal{G}$ gives its crosspoint complexity and the maximum number of crosspoints that lie between an input and an output of $\mathcal{G}$ gives its depth.

A graph $\mathcal{G}=(I, O, E)$ is called a sparse crossbar if each crosspoint in $E$ lies directly between an input in $I$ and an output in $O$. A sparse crossbar is called a binomial $\left(\left(\begin{array}{c}m \\ v\end{array}\right), m\right)$-network if every one of its $\left(\begin{array}{c}m \\ v\end{array}\right)$ inputs is connected to a distinct subset of $v$ outputs among its $m$ outputs. (See Figure 1). The number of outputs (inputs) to 
which an input (output) is connected is called its fanout (fanin), and the maximum number of outputs (inputs) to which an input (output) in $\mathcal{G}$ is connected is called the fanout (fanin) of $\mathcal{G}$.

The problems we consider in the paper deal with proving that certain sparse crossbars exhibit a concentrator behavior and with computing the minimum number of crosspoints it takes to construct a concentrator. Admittedly, the sparse crossbars we consider are to have as few crosspoints as possible. A secondary objective is to keep the fanout of the inputs and fanin of the outputs as small as possible and nearly constant over the entire set of inputs and outputs.

To prove that a sparse crossbar $\mathcal{G}=(I, O, E)$ is an $(n, m, c)$-concentrator, one will need to show that there exists a matching between every $c$ inputs in $I$ and some $c$ outputs in $O$. That is, for every $x_{1}, x_{2}, \ldots, x_{c} \in I$, one must show that there exist $y_{1}, y_{2}, \ldots, y_{r} \in O$ such that $\left(x_{1}, y_{1}\right),\left(x_{2}, y_{2}\right), \ldots,\left(x_{c}, y_{c}\right)$ constitute crosspoints in $E$. For example, in the binomial network in Figure 1(a), inputs 1,2,3 can be matched with outputs $1,2,3$ by the shaded crosspoints, and to prove that this sparse crossbar is a $(4,4,3)$-concentrator, we must show that such a matching exists between every three inputs and some three outputs. While, it is possible to exhaustively test this condition in this case, it is impractical to do so for even relatively small values of $n, m$, and $c$. For example, for $n=50, m=25$ and $c=25$, we will need to check $50 ! /(25 ! 25 !) \approx 1.27 \times 10^{14}$ cases. Obviously, it is not just impractical but impossible to verify that a sparse crossbar with $n$ inputs and $m$ outputs is an $(n, m, c)$-concentrator when $n$ and $m$ are not specified. In this case, we must resort to some formal argument that shows that $\mathcal{G}$ is an $(n, m, c)$-concentrator for all values of $n, m$ and $c$. In this connection, the following well-known theorem due to P. Hall will prove invaluable [6].

Theorem 1 (P. Hall) Let $O$ be a finite set and let $Y_{1}, Y_{2}, \ldots, Y_{r}$ be arbitrary subsets of $O$. There exist distinct elements $y_{i} \in Y_{i}, 1 \leq i \leq r$ if and only if the union of any $k$ of $Y_{1}, Y_{2}, \ldots, Y_{r}$ contains $k$ elements.

Hall's theorem will be invoked in our proofs as follows: To being with, the set $O$ in the theorem will denote the set of outputs of a sparse crossbar, $\mathcal{G}=(I, O, E)$, and $Y_{1}, Y_{2}, \ldots, Y_{r}$ will represent the subsets of all outputs in $O$ which are connected to some $r$ inputs $x_{1}, x_{2}, \ldots, x_{r}$ in $I$, in that order, i.e., $Y_{1}$ contains all the outputs connected to 
input $x_{1}, Y_{2}$ contains all the outputs connected to input $x_{2}$, and so on. The outputs in $Y_{i}$ are called the neighbors of $x_{i}$, and $Y_{i}$ is called the neighbor set of $x_{i}, 1 \leq i \leq r$. With this set up, we will attempt to establish that the union of $Y_{1}, Y_{2}, \ldots, Y_{r}$ contains at least $r$ outputs for any choices of $x_{1}, x_{2}, \ldots, x_{r}$ in $I$, and any $r, 1 \leq r \leq c$, where $c$ is the projected capacity of $\mathcal{G}$. This fact will then be used together with Hall's theorem to conclude that $\mathcal{G}$ is an $(n, m, c)$-concentrator. For example, it is easily verified that every input of the sparse crossbar in Figure 1(a) has three neighbors, and every two, three, or four inputs have at least four neighbors. Therefore, by Hall's theorem, this sparse crossbar is a $(4,4)$-concentrator. Our proofs will extend this example to sparse crossbars with any number of inputs and any number of outputs.

As for computing lower bounds on the crosspoint complexity of sparse crosbar concentrators, our proofs will rely on some elementary observations concerning the minimum number of neighbors of every subset of outputs with a certain cardinality must have. This minimum will be coupled with the required capacity of the sparse crossbar in question.

\section{$3 \quad$ Full Capacity Concentrators}

Nakamura and Masson[10] derived a lower bound on the crosspoint complexity of full capacity sparse crossbar concentrators, and proved that this bound is tight for a binomial $\left(\left(\begin{array}{c}m \\ m-2\end{array}\right), m\right)$-network for any $m \geq 2$. In this section we extend this result in two directions. First, we describe a new full capacity sparse crossbar concentrator whose crosspoint complexity matches the same lower bound for any number of inputs and any number of outputs. In contrast, the binomial $\left(\left(\begin{array}{c}m \\ 2\end{array}\right), m\right)$-network dictates a quadratic relation between its number of inputs and number of outputs, forcing their ratio to 0 as $m \rightarrow \infty$. Another problem with the binomial $\left(\left(\begin{array}{c}m \\ 2\end{array}\right), m\right)$-network is that its fanin (which is $m-2$ ) and fanout (which is $(m-1)(m-2) / 2)$ are very close to its number of inputs and number of outputs. Our second result in this section is a sparse crossbar $(2 m, m)$-concentrator construction that is optimal with respect to its crosspoint complexity, and has fanout which is nearly half the number of its outputs and fanin which is also nearly half of its inputs.

Before, we present these results, let us first recall the following facts from [10]. 
Theorem 2 (Nakamura-Masson) : Any sparse crossbar $(n, m)$-concentrator requires at least $m(n-m+1)$ crosspoints.

Corollary 1 (Nakamura-Masson) : For all $m \geq 3$, the binomial $\left(\left(\begin{array}{c}m \\ m-2\end{array}\right), m\right)$ - network yields a full capacity concentrator with a minimum number of crosspoints.

Corollary 1 shows that the lower bound of Theorem 1 is tight, but only for sparse crossbars with certain numbers of inputs and outputs. Our first result relaxes this constraint by a new concentrator construction, called a fat-and-slim crossbar.

Definition 1 : Let $\mathcal{G}=(I, O, E)$ be a sparse crossbar with $n$ inputs and $m$ outputs. Suppose that $I$ is partitioned into two sets $I_{1}$ and $I_{2}$, where $\left|I_{1}\right|=n-m$ and $\left|I_{2}\right|=m . \mathcal{G}$ is called an $(n, m)$-fat-and-slim crossbar if each of the $n-m$ inputs in $I_{1}$ is connected to all the $m$ outputs, and if each of the $m$ inputs in $I_{2}$ is connected to a single but distinct output.

Theorem 3 : For any positive integers $m$ and $n, m \leq n$, every $(n, m)$-fat-and-slim crossbar yields an $(n, m)$-concentrator with a minimum number of crosspoints.

Proof: Let $\mathcal{G}=(I, O, E)$ be an $(n, m)$-fat-and-slim crossbar, and $X=\left\{x_{1}, x_{2}, \ldots, x_{r}\right\}$ be an arbitrary $r$-subset of $\mathrm{I}$, where $1 \leq r \leq m$, and let $Y_{i}$ be the neighbor set of input $x_{i}, 1 \leq i \leq r$. By the construction of $\mathcal{G}$, it is obvious that if at least one of $x_{1}, x_{2}, \ldots x_{r}$ belongs to $I_{1}$ then $Y_{1} \cup Y_{2} \cup \ldots \cup Y_{r}$ contains at least $m$ outputs. On the other hand, if all of $x_{1}, x_{2}, \ldots x_{r}$ belong to $I_{2}$ then $Y_{1} \cup Y_{2} \cup \ldots \cup Y_{r}$ contains exactly $r$ outputs. Since this is true for any $r, 1 \leq r \leq m$, by Hall's theorem, there exist distinct outputs $y_{1}, y_{2}, \ldots, y_{m}$ such that $\left(1, y_{1}\right),\left(2, y_{2}\right), \ldots,\left(m, y_{m}\right)$ form crosspoints in $E$, and hence $\mathcal{G}$ is an $(n, m)$-concentrator. Furthermore, connecting each of the $n-m$ inputs of $\mathcal{G}$ to all its $m$ outputs requires $m(n-m)$ crosspoints and connecting the remaining $m$ inputs to the $m$ outputs requires $m$ crosspoints. Hence, $\mathcal{G}$ requires a total of $m(n-m)+m=m(n-m+1)$ crosspoints, matching the lower bound in Theorem 2. \|

Figure 2 shows a fat-and-slim $\operatorname{crossbar}^{1}$ for $n=13$ and $m=4$. In this particular

\footnotetext{
${ }^{1}$ The name fat-and-slim crossbar for this construction is coined not so much to capture its topology (i.e., its fat and slim sections), but rather to point out that while the fat-and-slim crossbar has the illusion of having too many crosspoints, in reality, its crosspoint complexity matches the lower bound in Theorem 2.
} 


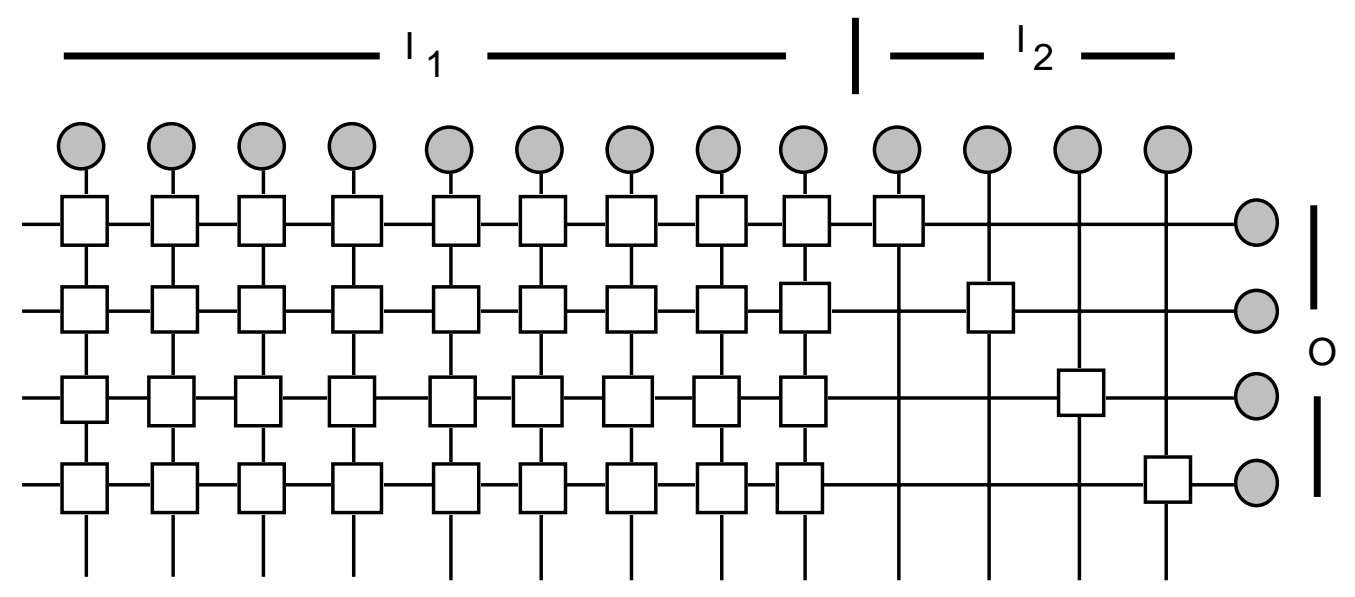

Fig. 2: A $(13,4)$-fat-and-slim crossbar with a minimum number of crosspoints.

fat-and-slim crossbar construction, the inputs in $I_{2}$ are connnected to the four outputs in a diagonal fashion. This is one of 4 ! possible constructions that can be obtained by permuting the inputs in $I_{2}$ onto the 4 outputs in 4 ! ways. In general, there are $\left(\begin{array}{c}n \\ m\end{array}\right) m !=n ! /(n-m)$ ! fat-and-slim crossbars with $n$ inputs and $m$ outputs.

This construction removes the restriction imposed by the binomial $\left(\left(\begin{array}{c}m \\ m-2\end{array}\right), m\right)$ - network on the number of inputs and outputs, but the distribution of its crosspoints over its inputs is not uniform even though each output has the same fanin. While we do not have a construction that solves this nonuniform fanout problem of the fat-andslim crossbars for arbitrary $n$ and $m$, our next construction settles the question when $n=2 m$ with a variance of 1 in the fanouts of the inputs, while keeping the fanin of the outputs fixed.

Theorem 4 : Let $\mathcal{G}=(I, O, E)$ be a sparse crossbar with $2 m$ inputs and $m$ outputs. Let $O=\{1,2,3, \ldots, m\}$, and suppose that $I$ is partitioned into two sets $I_{1}=$ $\{1,2,3, \ldots, m\}$ and $I_{2}=\{m+1, m+2, \ldots, 2 m\}$, where $\left|I_{1}\right|=\left|I_{2}\right|=m$. Suppose each input in $I_{1}$ is connected to all the odd outputs, and also input $2 i$ is connected to output $2 i, 1 \leq i \leq\lfloor m / 2\rfloor$. Likewise, suppose each input in $I_{2}$ is connected to the even outputs, and also input $2 i+1$ is connected to output $2(i-\lfloor m\rfloor)+1,\lfloor m / 2\rfloor \leq i \leq m-1$ (See Figure 3). $\mathcal{G}$ is a $(2 m, m)$-concentrator with a minimum number of crosspoints, fanout $=\lceil m / 2\rceil+1$ and fanin $=m+1$.

Proof: The proof of this theorem is similar to the proof of the previous theorem, but requires a more careful inspection of the neighbors of the inputs of $\mathcal{G}$. Let $X$ be an arbitrary $r$-subset of inputs, where $1 \leq r \leq m$, and let $X_{1}=X \cap I_{1}=\left\{x_{1}, x_{2}, \ldots, x_{p}\right\}$ 


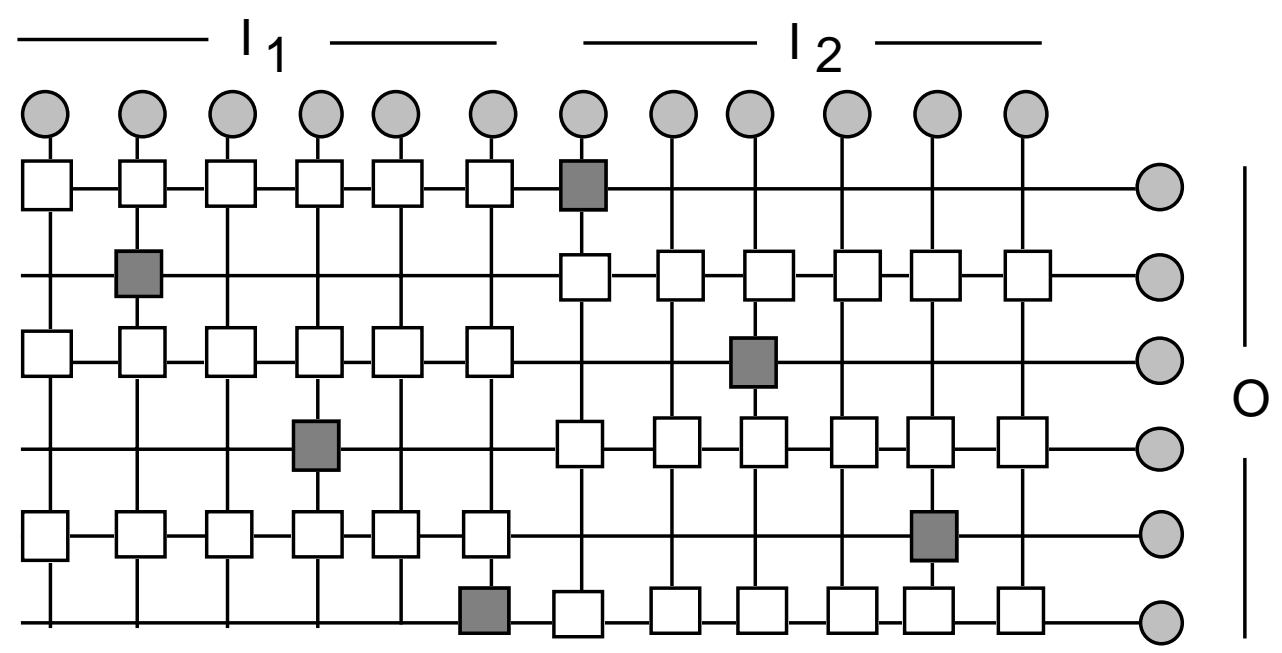

Fig. 3: A $(12,6)$-concentrator with a minimum number of crosspoints.

and $X_{2}=X \cap I_{2}=\left\{x_{1}^{\prime}, x_{2}^{\prime}, \ldots, x_{q}^{\prime}\right\}$, where $r=p+q$. Let $Y_{i}$ be the neighbor set of input $x_{i}, 1 \leq i \leq p$, and let $Y_{i}^{\prime}$ be the neighbor set of input $x_{i}^{\prime}, 1 \leq i \leq q$. It is easy to see that $Y_{1} \cup Y_{2} \cup \ldots \cup Y_{p}$ contains at least $\lceil m / 2\rceil+\epsilon_{1}$ outputs, where the first term in the sum accounts for the odd numbered neighbors of the inputs in $X \cap I_{1}$, and $\epsilon_{1} \geq 0$ accounts for the even numbered neighbors of the same inputs. Likewise, $Y_{1}^{\prime} \cup Y_{2}^{\prime} \cup \ldots \cup Y_{q}^{\prime}$ contains at least $\lfloor m / 2\rfloor+\epsilon_{2}$ outputs, where the first term in the sum accounts for the even numbered neighbors of the inputs in $X \cap I_{2}$, and $\epsilon_{2} \geq 0$ accounts for the odd numbered neighbors of the same inputs. Now, let

$$
Y=Y_{1} \cup Y_{2} \cup \ldots \cup Y_{p}
$$

and

$$
Y^{\prime}=Y_{1}^{\prime} \cup Y_{2}^{\prime} \cup \ldots \cup Y_{q}^{\prime}
$$

Then the number of neighbors of the inputs in $X$ is given by

$$
|Y|+\left|Y^{\prime}\right|-\left|Y \cap Y^{\prime}\right|
$$

Furthermore, the indices of the outputs in $Y$ and $Y^{\prime}$ show that the intersection of the two sets contains no more than

$$
\operatorname{Min}\left\{\epsilon_{1}, m / 2\right\}+\operatorname{Min}\left\{\epsilon_{2}, m / 2\right\}=\epsilon_{1}+\epsilon_{2}
$$

outputs. Therefore, the inputs in $X$ must have at least

$$
\lceil m / 2\rceil+\epsilon_{1}+\lfloor m / 2\rfloor+\epsilon_{2}-\left(\epsilon_{1}+\epsilon_{2}\right)=m \geq r
$$




\begin{tabular}{|l|c|c|c|c|}
\hline Concentrator & Crosspoint Comp. & Fanin & Fanout & I/O Restriction \\
\hline Binomial network & $m(n-m+1)$ & $n-m+1$ & $m-2$ & Yes \\
\hline Fat-and-Slim Crossbar & $m(n-m+1)$ & $n-m+1$ & $m$ & No \\
\hline Sparse Crossbar (Theorem 4) & $m(n-m+1)$ & $n-m+1$ & $(m / 2)+1$ & Yes \\
\hline
\end{tabular}

Table 1: Comparison of three sparse crossbar full capacity concentrators.

neighbors. Hence, by Hall's theorem, $\mathcal{G}$ is an $(n, m)$-concentrator. Furthermore, the number of crosspoints in this construction is given by $(m+1) m=(2 m-m+1) m$, and this matches the lower bound of Theorem 2 with $n=2 m$. The fanin and fanout expressions are obvious. \|

It is possible to extend this sparse crossbar concentrator construction to sparse crossbars with other input and output profiles. For example, if $n=k m$, where $k \geq 3$ is an integer, then a full capacity sparse crossbar concentrator can be obtained by connecting the first $2 m$ inputs to the $m$ outputs using this same sparse crossbar construction, and then connecting each of the remaining $k m-2 m=(k-2) m$ inputs to all the $m$ outputs. It is easy to show that this construction yields a full capacity concentrator. Furthermore, it uses

$$
(2 m-m+1) m+(k-2) m^{2}=(k-1) m^{2}+m=(k m-m+1) m
$$

crosspoints, matching the lower bound of Theorem 2. The main drawback of this construction is that the fanout varies over its inputs. It remains open to construct a sparse crossbar $(n, m)$-concentrator with a minimum crosspoint complexity and with fixed fanin and fanout for any $n$ and $m \leq n$.

The main features of the two concentrator constructions described in this section along with the binomial network are summarized in Table 1. All three constructions are optimal with respect to their crosspoint complexity. The advantage of the fatand-slim crossbar over the other two crossbars is the fact that it does not place any restrictions on its number of inputs and outputs, whereas the advantage of the sparse crossbar described in Theorem 4 is its relatively small fanout. 


\section{Bounded Capacity Concentrators}

The lower bound stated in Theorem 2 applies only to full capacity sparse crossbar concentrators. In this section, we consider the extension of this result to bounded capacity concentrators.

\section{A. Nakamura-Masson's Lower Bound}

We first recall the lower bound established in [10].

Theorem 5 (Nakamura-Masson) Any sparse crossbar $(n, m, c)$-concentrator requires $n x$ crosspoints where $x$ satisfies

$$
\frac{\left(\begin{array}{l}
c \\
x
\end{array}\right)}{\left(\begin{array}{l}
m \\
x
\end{array}\right)} n(c-x)-c^{2}+c=0
$$

For some values of $n, m$ and $c$, this bound is tight. In particular, the following holds.

Corollary 2 (Nakamura-Masson) For all $m, v \geq 2$, the binomial $\left(\left(\begin{array}{c}m \\ v\end{array}\right), m\right)$ network is a sparse crossbar $\left(\left(\begin{array}{c}m \\ v\end{array}\right), m, v+2\right)$-concentrator with a minimum number of crosspoints.

Remark 1 We note that the Nakamura-Masson's lower bound is also tight for $c=1$ and $c=m .(c=1$ gives $n$ crosspoints and $c=m$ gives $m(n-m+1)$ crosspoints both of which are clearly upper bounds as well, the latter by Theorem 3.) II

Except these three cases, it is not known if Nakamura-Masson's lower bound is also tight for other bounded capacity concentrators. Part of the difficulty stems from the implicit nature of the lower bound since one must solve $x$ in Eqn. 1 before the lower bound can be determined. When simplified, the expression on the left hand side gives a polynomial of degree $m-c+1$ in $x$ which is cumbersome to solve for an exact value of $x$ especially if $m>c$. Instead, Nakamura and Masson worked out a lower bound on $x$ which is given by

$$
x \geq c-\left(\begin{array}{c}
m \\
c
\end{array}\right) \frac{c(c-1)}{n} .
$$

Combining this lower bound on $x$ with Theorem 5 shows that any $(n, m, c)$-concentrator requires

$$
n x \geq n c-\left(\begin{array}{c}
m \\
c
\end{array}\right) c(c-1)
$$


crosspoints. If $m$ and $c$ are fixed, this lower bound approaches $n c$ as $n \rightarrow \infty$.

\section{B. The New Lower Bound}

The asymptotic lower bound given in Eqn. 3 is useful when $n>>m$, but, in most cases, one would be more interested in $(n, m)$-concentrators, where $m$ scales with $n$. In this case, this lower bound is negative for all $c \geq(n / m)+1$, and $m>>c$. This can be seen by noting that $\left(\begin{array}{c}m \\ c\end{array}\right) \geq m$, for $m>>c$, and hence

$$
n c-\left(\begin{array}{c}
m \\
c
\end{array}\right) c(c-1) \leq c(n-m(c-1)) \leq 0
$$

for all $c \geq(n / m)+1$.

Our next result gives a new lower bound on the crosspoint complexity of $(n, m, c)$ concentrators which is very easy to compute and closely follows Nakamura-Masson's exact lower bound for all $m=O(n)$.

Theorem 6 Any sparse crossbar $(n, m, c)$-concentrator requires $\left\lceil\frac{m(n-c+1)}{m-c+1}\right\rceil$ crosspoints.

Proof: Let $\mathcal{G}=(I, O, E)$ be a sparse crossbar $(n, m, c)$-concentrator. Then each $(m-c+1)$-subset of outputs in $O$ should be connected to at least $n-c+1$ inputs in $I$, since, otherwise, there will exist some $c$ inputs that are connected only to $c-1$ outputs $^{2}$, contradicting the fact that $\mathcal{G}$ is an $(n, m, c)$-concentrator. Let $P_{m-c+1}(O)$ denote the collection of all $(m-c+1)$-subsets of $O, d_{i}$ denote the fanin of output $i, 1 \leq i \leq m$, and $\rho_{i}$ denote the number of $(m-c+1)$-subsets of $O$ that contain output $i$. Since the number of neighbors (inputs) of the outputs in an $(m-c+1)$-subset of $O$ cannot be larger than the sum of the crosspoints connected to the outputs in that subset, we have

$$
\sum_{Y \in P_{m-c+1}(O)} \sum_{i \in Y} d_{i} \geq\left(\begin{array}{c}
m \\
m-c+1
\end{array}\right)(n-c+1)
$$

or equivalently,

$$
\sum_{i=1}^{m} \rho_{i} d_{i} \geq\left(\begin{array}{c}
m \\
m-c+1
\end{array}\right)(n-c+1)
$$

\footnotetext{
${ }^{2} \mathrm{~A}$ subset of outputs, $Y$, is said to be connected to a subset of input, $X$, if there exists a crosspoint between every input in $X$ and some output in $Y$.
} 
where the expressions on the left hand side of both inequalities sum the in-degrees of the outputs in $O$ over all of its $(m-c+1)$-subsets. Now, let $\kappa_{\mathcal{G}}(n, m, c)$ denote the number of crosspoints in $\mathcal{G}$. Noting that $\rho_{i}=\left(\begin{array}{c}m-1 \\ m-c\end{array}\right)$ and $\kappa_{\mathcal{G}}(n, m, c)=\sum_{i=1}^{m} d_{i}$, Eqn. 5 gives

$$
\left(\begin{array}{l}
m-1 \\
m-c
\end{array}\right) \kappa_{\mathcal{G}}(n, m, c) \geq\left(\begin{array}{c}
m \\
m-c+1
\end{array}\right)(n-c+1),
$$

or,

$$
\kappa_{\mathcal{G}}(n, m, c) \geq\left\lceil\frac{\left(\begin{array}{c}
m \\
m-c+1
\end{array}\right)(n-c+1)}{\left(\begin{array}{l}
m-1 \\
m-c
\end{array}\right)}\right\rceil
$$

which reduces to $\kappa_{\mathcal{G}}(n, m, c) \geq\left\lceil\frac{m(n-c+1)}{m-c+1}\right\rceil$ when simplified. $\quad \|$

An example may help clarify the derivation of this lower bound. Let $n=7, m=6$, and $c=2$. The lower bound is based on the fact that every $m-c+1=5$ outputs should be connected to at least $n-c+1=6$ inputs. If any 5 outputs are connected to fewer than 6 inputs then some two inputs can only be connected to at most 1 output, disqualifying the sparse crossbar in question from being a $(7,6,2)$-concentrator. With this requirement in place, Eqn. 4 states that the sum of the in-degrees of the outputs in any sparse $(7,6,2)$-concentrator over all 5 -subsets of its outputs must be $\geq\left(\begin{array}{c}6 \\ 6-2+1\end{array}\right)(7-2+1)=36$. The sum, in this case, is given by

$$
\begin{array}{rr} 
& d_{1}+d_{2}+d_{3}+d_{4}+d_{5} \\
+ & d_{1}+d_{2}+d_{3}+d_{4}+d_{6} \\
+ & d_{1}+d_{2}+d_{3}+d_{6}+d_{5} \\
+ & d_{1}+d_{2}+d_{6}+d_{4}+d_{5} \\
+ & d_{1}+d_{6}+d_{3}+d_{4}+d_{5} \\
+ & d_{6}+d_{2}+d_{3}+d_{4}+d_{5} .
\end{array}
$$

This simplifies to $5\left(d_{1}+d_{2}+d_{3}+d_{4}+d_{5}+d_{6}\right)$ (the sum on the left hand side of Eqn. 5) where 5 is the value of $\rho_{i}, 1 \leq i \leq 6$, i.e., the number of 5 -subsets of outputs that contain each of the six outputs. So, the lower bound in this case is $\left\lceil\left(\begin{array}{l}6 \\ 5\end{array}\right)(7-2+1) /\left(\begin{array}{l}6-1 \\ 6-2\end{array}\right)\right\rceil=$ $\lceil 36 / 5\rceil=8$.

We note that, as with Nakamura-Masson's exact lower bound, this new lower bound reduces to the lower bound given in Theorem 3 when $c=m$, and to $n$ when $c=1$. 
Figure 4 shows how the two bounds are related together for various other values of $c$. It should be pointed out that the new lower bound gets closer to NakamuraMasson's lower bound as $n / m \rightarrow 1$. The exact values of the two bounds are given in the Appendix for $n=100, m=90,1 \leq c \leq 90$, and $n=100, m=50,1 \leq c \leq 50$. We note that computing the exact values of Nakamura-Masson lower bound is much more time consuming than computing new lower bound as the former requires solving a polynomial of degree $m-c+1$.

\section{Bounded Capacity Sparse Crossbar Concentrators}

At this point it is reasonable to ask whether we can construct a bounded capacity sparse crossbar $(n, m, c)$-concentrator for any $n, m$, and $c$ with a minimum crosspoint complexity. Unlike the full capacity case, the resolution of this question is complicated by two related facts. First, the new lower bound we derived in the previous section is not as tight as Nakamura-Masson's lower bound. Second, Nakamura-Masson's lower bound is not explicit enough to suggest a bounded capacity sparse crossbar concentrator construction whose crosspoint complexity may somehow match it by a constant factor.

Given this reality, we present in this section a bounded capacity sparse crossbar concentrator whose crosspoint complexity comes within a constant factor of the new lower bound, when $m \leq n \leq m+\sqrt{m}$, and $n-m \leq c \leq \sqrt{m}$. A similar construction for other values of $n, m$ and $c$ remains an open problem.

Theorem 7 Let $\mathcal{G}=(I, O, E)$ be a sparse crossbar, where $m \leq n \leq m+\sqrt{m}$, $n-m \leq c \leq \sqrt{m}$. Suppose that the inputs in $I$ are partitioned into two sets $I_{1}=$ $\{1,2, \ldots, n-c\}$, and $I_{2}=\{n-c+1, n-c+2, \ldots, n\}$, and the outputs in $O$ are partitioned into $\lceil\mathrm{m} / \mathrm{c}\rceil$ sets, $O_{i}=\{(i-1) c+1,(i-1) c+2, \ldots, i c\}, 1 \leq i \leq\lfloor\mathrm{m} / \mathrm{c}\rfloor$, and $O_{\lceil m / c\rceil}=\{\lfloor m / c\rfloor c,\lfloor m / c\rfloor c+1, \ldots, m\}$. Let the inputs in $I_{2}$ be connected to the outputs in $O_{1}, O_{2}, \ldots, O_{c}$ in a diagonal fashion, i.e., let $(n-c+j,(i-1) c+j) \in E, 1 \leq i \leq$ $c, 1 \leq j \leq c$. Furthermore, let input $i$ in $I_{1}$ be connected to output $i$ in $O$. Then $\mathcal{G}$ (See Figure 5) is an $(n, m, c)$-concentrator with $m-c+c^{2}$ crosspoints.

Proof: We need to show that every subset of $c$ inputs can be matched with some $c$ outputs, $n-m \leq c \leq \sqrt{m}$. It is obvious from the construction that if these $c$ inputs 

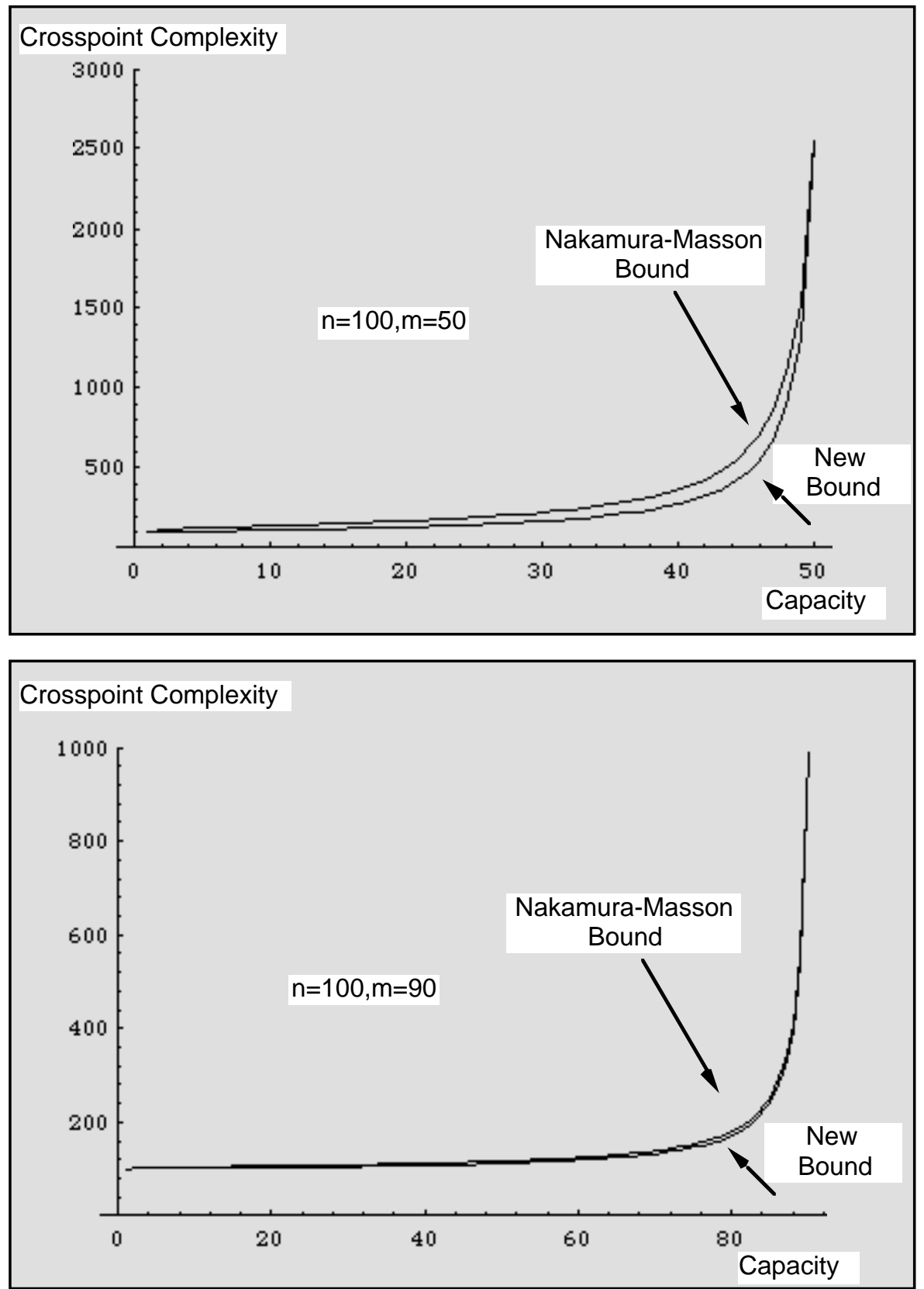

Fig. 4: Comparison of Nakamura-Masson's lower bound with the new lower bound. 
all belong to $I_{1}$ or all belong to $I_{2}$ then this can be done. So, consider an arbitrary but fixed set of $\alpha \leq c$ inputs, $X$, where $X_{1}=X \cap I_{1} \neq \emptyset$, and $X_{2}=X \cap I_{2} \neq \emptyset$. Let $\alpha_{1}=\left|X_{1}\right|, \alpha_{2}=\left|X_{2}\right|$, and let $Y_{1}$ and $Y_{2}$ be the sets of neighbors of $X_{1}$ and $X_{2}$ in $\mathcal{G}$. We have

$$
\left|Y_{1} \cup Y_{2}\right|=\left|Y_{1}\right|+\left|Y_{2}\right|-\left|Y_{1} \cap Y_{2}\right|,
$$

and by the construction of $\mathcal{G},\left|Y_{1}\right|=\alpha_{1},\left|Y_{2}\right|=\alpha_{2} c,\left|Y_{1} \cap Y_{2}\right| \leq \operatorname{Min}\left\{\alpha_{1}, \alpha_{2} c\right\}$ so that

$$
\left|Y_{1} \cup Y_{2}\right| \geq \alpha_{1}+\alpha_{2} c-\operatorname{Min}\left\{\alpha_{1}, \alpha_{2} c\right\}
$$

Now, if $\operatorname{Min}\left\{\alpha_{1}, \alpha_{2} c\right\}=\alpha_{1}$ then

$$
\left|Y_{1} \cup Y_{2}\right| \geq \alpha_{1}+\alpha_{2} c-\alpha_{1}=\alpha_{2} c
$$

and since $\alpha_{2} \geq 1$,

$$
\left|Y_{1} \cup Y_{2}\right| \geq \alpha_{2} c \geq c \geq \alpha
$$

On the other hand, if $\operatorname{Min}\left\{\alpha_{1}, \alpha_{2}\right\}=\alpha_{2} c$, then

$$
\left|Y_{1} \cup Y_{2}\right| \geq \alpha_{1}+\alpha_{2} c-\alpha_{2} c=\alpha_{1},
$$

but since $\alpha_{1} \geq \alpha_{2} c \geq c$, we again have

$$
\left|Y_{1} \cup Y_{2}\right| \geq c \geq \alpha
$$

We have thus shown that the set of neighbors of any $\alpha \leq c$ inputs contains at least $\alpha$ outputs. Hence by Hall's theorem, there must exist $\alpha$ distinct outputs to which any $\alpha \leq c$ inputs can be matched, and so $\mathcal{G}$ is an $(n, m, c)$-concentrator for any $c, n-m \leq$ $c \leq \sqrt{m}$. Moreover, its construction reveals that it encompasses $n-c+c^{2}$ crosspoints, where the first term accounts for the number of crosspoints connected to the inputs in $I_{1}$, while the second term accounts for the number of crosspoints connected to the inputs in $I_{2}$. $\|$

The crosspoint complexity of this sparse crossbar concentrator is quite close to the lower bound derived in the previous section. To see this, we note that

$$
n-c+c^{2} \leq(n-c) m /(m-c+1)+c^{2}
$$




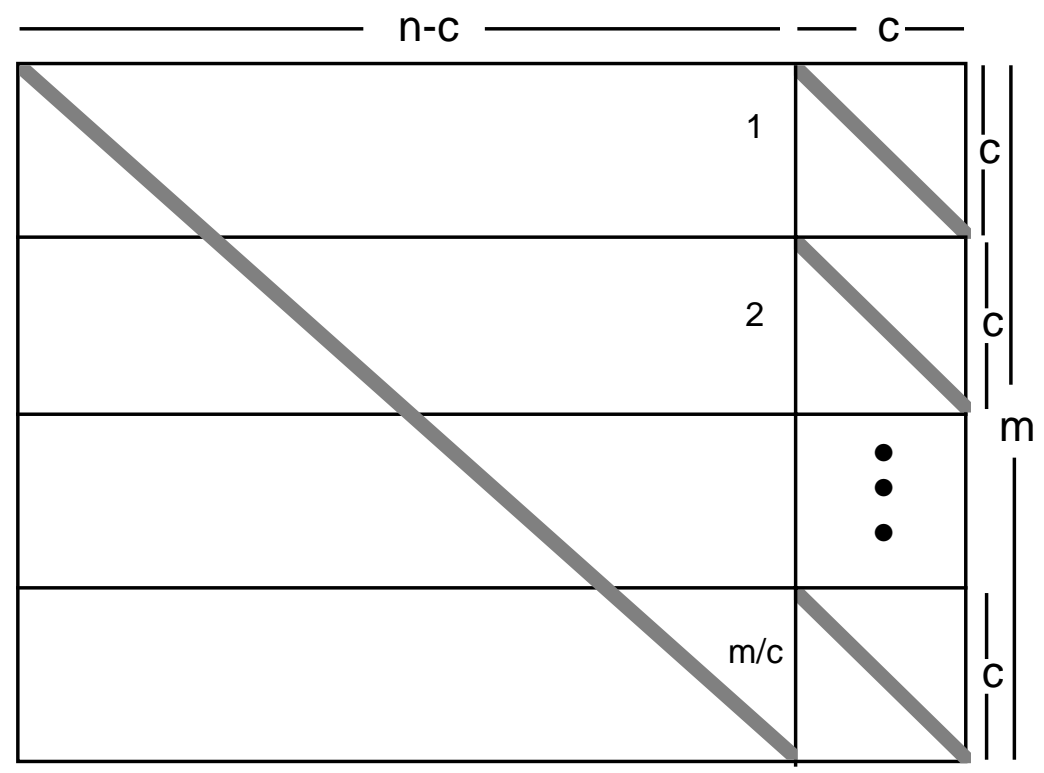

Fig. 5: The structure of the sparse crossbar construction in Theorem 7 when $c=m^{1 / 2}$, and $n-c=m$. (The gray lines indicate where the crosspoints are located.)

so that difference between the crosspoint complexity of this sparse crossbar concentrator and the lower bound is at most $c^{2}-m /(m-c+1) \leq m$. We also note that a more direct construction of a sparse crossbar $(n, m, c)$-concentrator obtained by connecting each input to some $c$ outputs yields a crosspoint complexity of $n c$ which gives $O\left(n^{1.5}\right)$ crosspoints as compared to $O(n)$ crosspoints of this construction for $n-m \leq c \leq \sqrt{m}$. We illustrate this bounded capacity sparse crossbar construction in Figure 5 for $n=$ $18, m=16$ and $c=4$. The shaded boxes on the left show how an input in $I_{2}$ can be blocked by as many as $c-1$ inputs in $I_{1}$, demostrating the necessity of $c$ crosspoints per each input in $I_{2}$. This particular construction uses $14+16=30$ crosspoints, whereas the lower bound yields $\lceil(n-c+1) m /(m-c+1)\rceil=\lceil 15 \times 16 / 13\rceil=20$ crosspoints. As another example, simple arithmetic shows that if $n=18, m=16$, and $c=2$, then the bounded capacity sparse crossbar concentrator of Theorem 7 requires 20 crosspoints whereas the lower bound of Theorem 6 gives 19 crosspoints.

\section{Crosspoint Complexity of Cascaded Sparse Crossbars}

The lower bound on the sparse crospoint complexity of sparse crossbar concentrators can be extended to cascades of sparse crossbars.

Let $\mathcal{G}(p, q)$ denote a sparse crossbar $(p, q)$-concentrator, where $p \geq q$, and let $\mathcal{G}(n, m, c$ : 


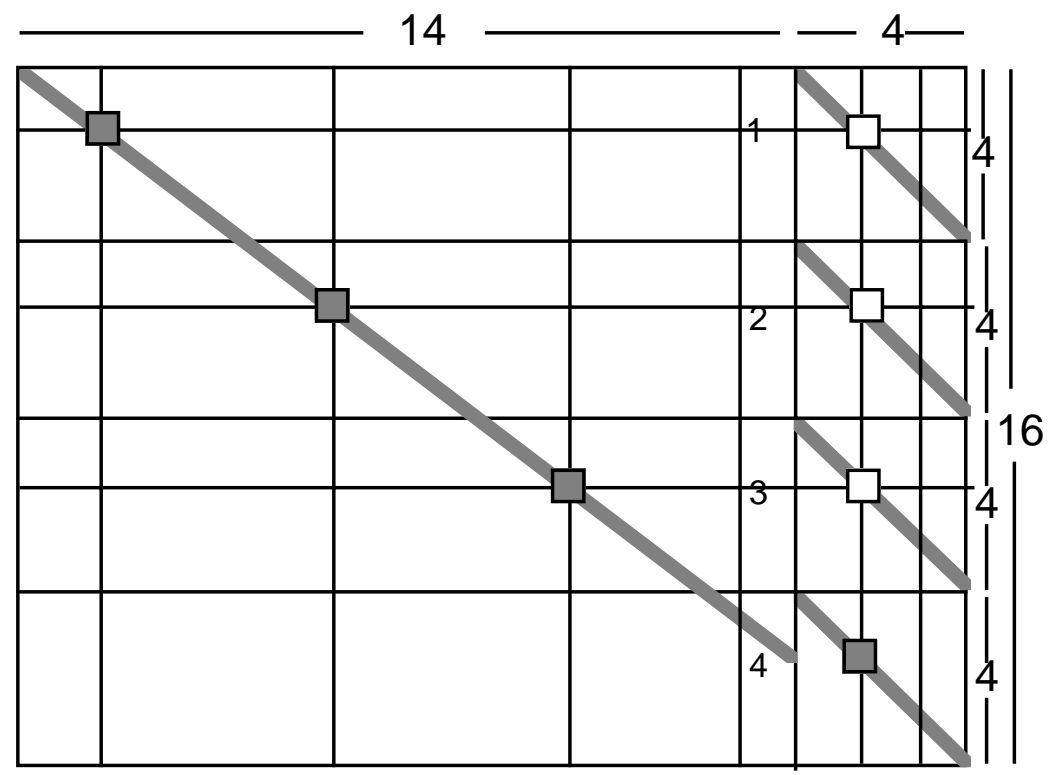

Fig. 6: Illustration of the sparse crossbar construction in Theorem 7 for $n=18$, $m=16$, and $c=4$.

$k)$ be an $(n, m, c)$-concentrator constructed by cascading $k$ sparse crossbar concentrators $\mathcal{G}_{1}\left(n=m_{0}, m_{1}\right), \mathcal{G}_{2}\left(m_{1}, m_{2}\right), \ldots, \mathcal{G}_{k}\left(m_{k-1}, m_{k}=m\right)$ as shown in Figure 7 . Since $\mathcal{G}(n, m, c)$ has capacity $c$, the capacity of each of the sparse crossbars in the cascade must at least be $c$. Furthermore, the crosspoint complexity of $\mathcal{G}(n, m, c: k)$ is given by the sum of the crosspoint complexities of all the sparse crossbars in the cascade. Combining these facts with the lower bound in Theorem 6 , we conclude that the crosspoint complexity, $\kappa_{\mathcal{G}}(n, m, c: k)$, of $\mathcal{G}(n, m, c: k)$ must satisfy the inequality

$$
\kappa_{\mathcal{G}}(n, m, c: k) \geq \sum_{i=1}^{k}\left\lceil\frac{m_{i}\left(m_{i-1}-c+1\right)}{m_{i}-c+1}\right\rceil .
$$

We use this fact to prove

\section{Theorem 8}

$$
\kappa_{\mathcal{G}}(n, m, c: k) \geq\left\lceil k \cdot m+(n-m)+(c-1) \cdot k \cdot\left(\left(\frac{n-c+1}{m-c+1}\right)^{1 / k}-1\right)\right\rceil .
$$

Proof: From Eqn. 8,

$$
\begin{aligned}
\kappa_{\mathcal{G}}(n, m, c: k) & \geq \sum_{i=1}^{k}\left\lceil m_{i}+\frac{m_{i} \cdot\left(m_{i-1}-m_{i}\right)}{m_{i}-c+1}\right\rceil \\
& \geq\left\lceil\sum_{i=1}^{k} m_{i}+\frac{m_{i} \cdot\left(m_{i-1}-m_{i}\right)}{m_{i}-c+1}\right\rceil
\end{aligned}
$$




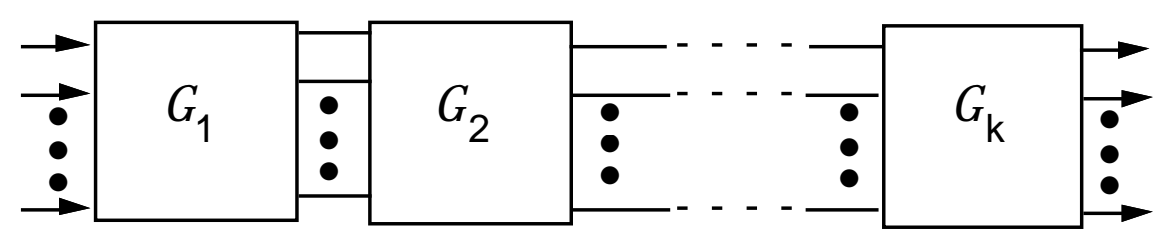

Fig. 7: Cascade of $k$ concentrators.

Let $\alpha=c-1$, and $\delta_{i}=m_{i-1}-m_{i}$. Then,

$$
\begin{aligned}
\sum_{i=1}^{k} m_{i}+\frac{m_{i} \cdot\left(m_{i-1}-m_{i}\right)}{m_{i}-c+1} & =\sum_{i=1}^{k} m_{i}+\sum_{i=1}^{k} \frac{m_{i} \cdot \delta_{i}}{m_{i}-\alpha} \\
& =\sum_{i=1}^{k} m_{i}+\sum_{i=1}^{k}\left(1+\frac{\alpha}{m_{i}-\alpha}\right) \cdot \delta_{i} \\
& =\sum_{i=1}^{k} m_{i}+\sum_{i=1}^{k} \delta_{i}+\sum_{i=1}^{k} \frac{\alpha}{m_{i}-\alpha} \cdot \delta_{i} .
\end{aligned}
$$

Now,

$$
\begin{aligned}
\sum_{i=1}^{k} \delta_{i} & =\left(m_{0}-m_{1}\right)+\left(m_{1}-m_{2}\right)+\ldots+\left(m_{k-1}-m_{k}\right) \\
& =m_{0}-m_{k} \\
& =n-m
\end{aligned}
$$

and

$$
\sum_{i=1}^{k} \frac{\alpha}{m_{i}-\alpha} \cdot \delta_{i}=\sum_{i=1}^{k} \frac{\alpha}{m_{i}-\alpha} \cdot\left(m_{i-1}-m_{i}\right)
$$

Let $m_{i}-\alpha=m \prime_{i}$, then Eqn. 16 becomes

$$
\begin{aligned}
\alpha \cdot \sum_{i=1}^{k} \frac{m \prime_{i-1}-m \prime_{i}}{m \prime_{i}} & =\alpha \cdot \sum_{i=1}^{k}\left(\frac{m \prime_{i-1}}{m \prime_{i}}-1\right) \\
& =\alpha \cdot\left(\left(\sum_{i=1}^{k} \frac{m \prime_{i-1}}{m \prime_{i}}\right)-k\right) .
\end{aligned}
$$

By the Arithmetic/Geometric inequality,

$$
\sum_{i=1}^{k} \frac{m \prime_{i-1}}{m \prime_{i}} \geq k \cdot\left(\frac{m \prime_{0}}{m \prime_{1}} \frac{m \prime_{1}}{m \prime_{2}} \ldots \frac{m \prime_{k-1}}{m \prime_{k}}\right)^{1 / k}
$$




$$
\begin{aligned}
& =k \cdot\left(\frac{m \prime_{0}}{m \prime_{k}}\right)^{1 / k} . \\
& =k \cdot\left(\frac{n-\alpha}{m-\alpha}\right)^{1 / k}
\end{aligned}
$$

This inequality combined with Eqn. 18 gives

$$
\alpha \cdot\left(\sum_{i=1}^{k} \frac{m \prime_{i-1}}{m \prime_{i}}-k\right) \geq \alpha \cdot\left(k \cdot\left(\frac{n-\alpha}{m-\alpha}\right)^{1 / k}-k\right) .
$$

Moreover, it is easily seen that

$$
\sum_{i=1}^{k} m_{i} \geq k \cdot m
$$

Combining Eqns. 14, 15, 19, and 20 together, and recalling that $\alpha=c-1$, we find

$$
\begin{aligned}
\sum_{i=1}^{k} m_{i}+\frac{m_{i} \cdot\left(m_{i-1}-m_{i}\right)}{m_{i}-c+1} \geq & k \cdot m+(n-m)+\alpha \cdot k \cdot\left(\left(\frac{n-\alpha}{m-\alpha}\right)^{1 / k}-1\right) \\
\geq & k \cdot m+(n-m)+(c-1) \cdot k \cdot \\
& \left(\left(\frac{n-c+1}{m-c+1}\right)^{1 / k}-1\right) .
\end{aligned}
$$

Therefore,

$$
\kappa_{\mathcal{G}}(n, m, c: k) \geq\left\lceil k \cdot m+(n-m)+(c-1) \cdot k \cdot\left(\left(\frac{n-c+1}{m-c+1}\right)^{1 / k}-1\right)\right\rceil,
$$

and the statement follows. $\|$

Remark 2: We note that this lower bound reduces to the lower bound in Theorem 6 for $k=1 . \quad \|$

\section{Concluding Remarks}

The three main results of this paper are (1) the construction of a full capacity sparse crossbar concentrator with a minimum number of crosspoints for any number inputs and any number of outputs, (2) new, easily computable lower bounds on the crosspoint complexities of bounded capacity sparse crossbar concentrators and cascades of bounded capacity sparse crossbar concentrators, and (3) the construction of a bounded capacity sparse crossbar concentrator with crosspoint complexity which is linear in its 
number of inputs (and outputs) when the capacity does not exceed the square root of the number of outputs. These results extend Nakamura-Masson's earlier work on sparse crossbar concentrators in a tangible way, while leaving out the construction of an $(n, m, c)$-concentrator with a crosspoint complexity which is within a constant factor of either of the lower bounds stated in Section 4 for arbitrary $n, m$, and $c$ as an open problem.

\section{References}

[1] N. Alon. Eigenvalues and expanders. Combinatorics, pp. 83-96, 1986.

[2] R. A. Brualdi and H. J. Ryser. Combinatorial Matrix Theory. Cambridge University Press, 1991.

[3] Minze V. Chien and A. Yavuz Oruç. High performance concentrators and superconcentrators using multiplexing schemes. IEEE Transactions on Communications, pp. 3045-3051, November 1994.

[4] O. Gabber and Z. Galil. Explicit constructions of linear sized superconcentrators. Journal of Computer and System Sciences, pp. 407-420, 1981.

[5] Chingyuh Jan and A. Yavuz Oruç. Fast self-routing permutation switching on an asymptotically minimum cost network. IEEE Transactions on Computers, pp. 1369-1379, December 1993.

[6] P. Hall. On representatives of subsets. Journal of London Mathematical Society, pp. 134-151, 1935.

[7] C. Y. Lee and A. Yavuz Oruç. Design of practical and easily routable generalized connectors. In Proc. International Conference on Parallel Processing, St. Charles, IL, August 1992, pp. I-138-I-141.

[8] G. A. Margulis. Explicit constructions of concentrators, Problems of Information Transmission, pp. 325-332, Vol. 9, No. 4,1973. 
[9] G.M. Masson. Binomial switching networks for concentration and distribution IEEE Transactions on Communications, 1977, September, Vol. COM-25, pp. 873883.

[10] S. Nakamura and G.M. Masson. Lower bounds on crosspoints in concentrators, IEEE Transactions on Computers 1982, Vol. C-31, December, pp. 1173-1178

[11] M. S. Pinsker. On the complexity of a concentrator In Proceedings of 7th International Teletraffic Congress, pp. 318/1-318/4, 1973.

[12] D. A. Tugal and O Tugal. Data Transmission. Mc.Graw Hill, New York 1989. 


\section{Appendix:}

\begin{tabular}{|c|l|c||c|c|c|}
\hline Capacity & NLB & N-MLB & Capacity & NLB & N-MLB \\
\hline 1 & 100 & 100 & 46 & 110 & 116 \\
2 & 101 & 103 & 47 & 111 & 116 \\
3 & 101 & 103 & 48 & 111 & 117 \\
4 & 101 & 103 & 49 & 112 & 117 \\
5 & 101 & 104 & 50 & 112 & 118 \\
6 & 101 & 104 & 51 & 113 & 118 \\
7 & 101 & 104 & 52 & 114 & 119 \\
8 & 101 & 105 & 53 & 114 & 120 \\
9 & 101 & 105 & 54 & 115 & 120 \\
10 & 102 & 105 & 55 & 115 & 121 \\
11 & 102 & 105 & 56 & 116 & 122 \\
12 & 102 & 105 & 57 & 117 & 123 \\
13 & 102 & 106 & 58 & 118 & 123 \\
14 & 102 & 106 & 59 & 119 & 124 \\
15 & 102 & 106 & 60 & 120 & 125 \\
16 & 102 & 106 & 61 & 120 & 126 \\
17 & 103 & 107 & 62 & 122 & 127 \\
18 & 103 & 107 & 63 & 123 & 129 \\
19 & 103 & 107 & 64 & 124 & 130 \\
20 & 103 & 107 & 65 & 125 & 131 \\
21 & 103 & 107 & 66 & 126 & 133 \\
22 & 104 & 108 & 67 & 128 & 134 \\
23 & 104 & 108 & 68 & 130 & 136 \\
24 & 104 & 108 & 69 & 131 & 138 \\
25 & 104 & 108 & 70 & 133 & 140 \\
26 & 104 & 109 & 71 & 134 & 142 \\
27 & 105 & 109 & 72 & 135 & 145 \\
28 & 105 & 109 & 73 & 148 & 147 \\
29 & 105 & 109 & 74 & 143 & 150 \\
30 & 105 & 110 & 75 & 147 & 154 \\
31 & 105 & 110 & 76 & 150 & 158 \\
32 & 106 & 110 & 77 & 155 & 162 \\
33 & 106 & 111 & 78 & 160 & 167 \\
34 & 106 & 111 & 79 & 165 & 173 \\
35 & 107 & 111 & 80 & 172 & 180 \\
36 & 107 & 112 & 81 & 180 & 189 \\
37 & 107 & 112 & 82 & 190 & 199 \\
38 & 107 & 112 & 83 & 203 & 212 \\
39 & 108 & 113 & 84 & 219 & 229 \\
40 & 108 & 113 & 85 & 240 & 251 \\
41 & 108 & 113 & 86 & 270 & 282 \\
42 & 109 & 114 & 87 & 315 & 328 \\
43 & 109 & 114 & 88 & 390 & 404 \\
44 & 110 & 115 & 89 & 540 & 555 \\
45 & 110 & 115 & 90 & 990 & 990 \\
\hline & & & & & \\
\hline
\end{tabular}

Exact Values of Nakamura-Masson and new lower bounds for $n=100, m=90$, and $c=1,2, \ldots 90$.

N-MLB: Nakamura-Masson Lower bound. NLB: New Lower Bound. 


\begin{tabular}{|c|l|l||c|c|c|}
\hline Capacity & NLB & N-MLB & Capacity & NLB & N-MLB \\
\hline 1 & 100 & 100 & 26 & 150 & 198 \\
2 & 102 & 116 & 27 & 155 & 203 \\
3 & 103 & 120 & 28 & 159 & 210 \\
4 & 104 & 123 & 29 & 164 & 217 \\
5 & 105 & 126 & 30 & 170 & 224 \\
6 & 106 & 129 & 31 & 175 & 231 \\
7 & 107 & 132 & 32 & 182 & 241 \\
8 & 109 & 134 & 33 & 189 & 251 \\
9 & 110 & 137 & 34 & 198 & 262 \\
10 & 111 & 139 & 35 & 207 & 274 \\
11 & 113 & 142 & 36 & 217 & 288 \\
12 & 115 & 145 & 37 & 229 & 304 \\
13 & 116 & 147 & 38 & 243 & 322 \\
14 & 118 & 150 & 39 & 259 & 343 \\
15 & 120 & 153 & 40 & 278 & 368 \\
16 & 122 & 156 & 41 & 300 & 397 \\
17 & 124 & 160 & 42 & 328 & 433 \\
18 & 126 & 163 & 43 & 363 & 477 \\
19 & 129 & 166 & 44 & 408 & 533 \\
20 & 131 & 170 & 45 & 467 & 607 \\
21 & 134 & 174 & 46 & 550 & 708 \\
22 & 137 & 178 & 47 & 675 & 856 \\
23 & 140 & 183 & 48 & 884 & 1090 \\
24 & 143 & 187 & 49 & 1300 & 1521 \\
25 & 147 & 192 & 50 & 2550 & 2550 \\
& & & & & \\
& & & & & \\
\end{tabular}

Exact Values of Nakamura-Masson and new lower bounds for $n=100, m=50$, and $c=1,2, \ldots 50$.

N-MLB: Nakamura-Masson Lower bound.

NLB: New Lower Bound. 